\title{
Corrigendum
}

\section{The effect of 12 weeks of euenergetic high-protein diet in regulating appetite and body composition of women with normal-weight obesity: a randomised controlled trial - Corrigendum}

\author{
Neda Haghighat, Damoon Ashtary-Larky, Reza Bagheri, Marzieh Mahmoodi, Majdadin Rajaei, \\ Meysam Alipour, Wesam Kooti, Vahideh Aghamohammdi and Alexei Wong
}

(First published online 09 June 2020)

DOI: https://doi.org/10.1017/S0007114520002019

We were made aware that the listed mean between-group difference and 95\% confidence interval (CI) for lean body mass (LBM), fat mass (FM), body fat percentage (BFP) and waist circumference (WC) at 12 weeks in the abstract and result sections appeared incorrectly reported, which was indeed the case.

Consequently, the original text in the abstract section "After 12 weeks, the LBM was higher in HP compared to no significant changes in the SP group [mean between-group difference $=1.5 \mathrm{~kg}$ (95\% confidence interval $\{C I\}, 3.1$ to 0.01, effect size $\{d\}=0.4)]$. Furthermore, the HP group had lower FM [mean between-group difference, -1.1 kg (95\% CI, 1 to -3.3; $d=-0.2)]$, body fat percentage (BFP) [mean between-group difference, $-2 \%$ (95\% CI, 0.7 to -5.2; $d=-0.3)$ ] and WC [mean between-group difference, $-1.4 \mathrm{~cm}(95 \%$ CI, 0.6 to -3.6; $d=-0.2)]$ at the end of the study in comparison to the SP group" was amended to include the correct information and now reads as follows: "After 12 weeks, LBM was higher in HP compared to no significant changes in the SP group [mean between-group difference $=1.30 \mathrm{~kg}(95 \%$ confidence interval $\{C I\}, 0.02$ to 2.58 , effect size $\{d\}=0.4)]$. Furthermore, the HP group had lower FM [mean between-group difference, $-1.37 \mathrm{~kg}(95 \% \mathrm{CI},-2.72$ to -0.03; $d=-0.2)]$, body fat percentage (BFP) [mean between-group difference, $-2.92 \%(95 \% C I,-5.70$ to $-0.14 ; d=-0.3)]$ and $W C[$ mean between-group difference, $-2.19 \mathrm{~cm}(95 \% C I,-3.53$ to $-0.85 ; d=-0.2)]$ at the end of the study in comparison to the SP group".

The original text in the results section "At 12 weeks, the HP group had a significantly $(p<0.05)$ greater increase in LBM than did the SP group [the mean between-group difference, $1.5 \mathrm{~kg}$ (95\% confidence interval \{ $C I\}, 3.1$ to $0.01, d: 0.4)]$. There were significantly ( $p<0.05$ ) greater declines in FM in the HP than in the SP group [mean between-group difference, $-1.1 \mathrm{~kg}$ (95\% CI, 1 to -3.3; d:-O.2)]. The change from baseline to 12 weeks in BFP also differed significantly between the two groups [mean between-group difference, $-2 \%$ (95\% CI, 0.7 to -5.2; d:-0.3); $p<0.05]$. In addition, the HP group had significantly larger declines in WC than the SP group [mean between-group difference, $-1.4 \mathrm{~cm}$ (95\% CI, 0.6 to - 3.6; $d:-0.2) ; p<0.05$ ]" was also amended to incorporate the accurate information and now reads as follows: "At 12 weeks, the HP group had a significant $(p<0.05)$ increase in LBM compared to no changes in the SP group [mean between-group difference, $1.30 \mathrm{~kg}$ (95\% confidence interval $\{C I\}, 0.02$ to 2.58 , effect size $\{d\}=0.4)]$. There were also significant $(p<0.05)$ declines in FM [mean between-group difference, $-1.37 \mathrm{~kg}(95 \% \mathrm{CI},-2.72$ to $-0.03 ; d=-0.2)]$ and BFP [mean betweengroup difference, $-2.92 \%$ (95\% CI, -5.70 to $-0.14 ; d=-0.3)]$ in the HP compared to no changes in the SP group. In addition, the HP group had significantly lower WC than the SP group [mean between-group difference, $-2.19 \mathrm{~cm}(95 \% \mathrm{CI},-3.53$ to -0.85 ; $d=-0.2) ; p<0.05]$ after 12 weeks." 